Related content

The connection between Dirac dynamic and parity symmetry

C. H. Coronado Villalobos and R. J. Bueno Rogerio

Spin-half fermions with mass dimension one: theory, phenomenology, and darkmatter

D V Ahluwalia-Khalilova and D Grumiller

Evading Weinberg's no-go theorem to construct mass dimension one fermions: Constructing darkness Dharam Vir Ahluwalia 


\title{
Non-standard Dirac adjoint spinor: The emergence of a new dual
}

\author{
R. J. Bueno Rogerio ${ }^{1}$ and C. H. Coronado Villalobos ${ }^{2}$ \\ 1 Universidade Estadual Paulista (Unesp), Faculdade de Engenharia, Guaratinguetá, \\ Departamento de Física e Química - 12516-410, Guaratinguetá, SP, Brazil \\ 2 Instituto de Fsica, Universidade Federal Fluminense (UFF) - 24210-346, Niteri, RJ, Brazil
}

received 21 November 2017; accepted in final form 2 March 2018

published online 20 March 2018

PACS 11.10.-z - Field theory

PACS $03.70 .+k$ - Theory of quantized fields

PACS 03.65.Fd - Algebraic methods

\begin{abstract}
In this present communication we provide a new derivation of the Dirac dual structure by employing a different approach from the originally proposed one. Following a general and rigorous mathematical process to compute the dual structure, we investigate if is possible to break the existing "rigidity" in its primordial formulation. For this task, firstly, we look towards to understand the core of the Dirac spinors construction (Dirac P. A. M., Proc. R. Soc. Lond. $A, 117$ (1928) 610). and then, we suggest to build an alternative dual structure for the Dirac spinor, which preserves an invariant norm under any $S L(2, \mathcal{C})$ transformation. Finally, we verify if the prominent physical content is maintained or if it is affected by such construction.

Copyright (C) EPLA, 2018
\end{abstract}

Introduction. - Commonly in textbooks the two problems that the Klein-Gordon equation suffers are presented: the probability density being not positive definite, and states of negative energy that occur. These problems arise due to the interpretation of the Klein-Gordon equation as a single-particle equation of a given wave function. Dirac's insight was to look towards a first-order equation (field equation), aiming to circumvent the mentioned problems. The solutions of Dirac equation, namely Dirac spinors, cannot be interpreted as wave functions due to the fact that they do not transform from one frame to another unitarily, for this reason we are led to the second quantization (which has an interpretation as a many-particle theory), and then we arrive at a successful particle interpretation and the problems associated with the KleinGordon equation are bypassed.

As is well known, much of the physics associated to spinor fields is unveiled from its bilinear covariants for the simple reason that single fermions are not directly experienced [1]. As a useful exercise to the reader, we must remember that a single spinor does not have a usual transformation under a complete rotation, i.e., $\psi \stackrel{2 \pi}{\rightarrow}-\psi$, thus, to be physically and mathematically coherent, we need to define a structure that, when combined with the spinor $\psi$, provides $\bar{\psi} \psi \stackrel{2 \pi}{\rightarrow} \bar{\psi} \psi$, where $\bar{\psi}$ is called by adjoint, note that the dual along with the usual spinor structure bring us relevant physical information. So, in view of the foregoing, Dirac solved the problem of the probability density being not positive definite, by using the solutions of his first-order differential equation, thus, the current density defined as $J^{\mu}=\bar{\psi} \gamma^{\mu} \psi$, where $\bar{\psi}=\psi^{\dagger} \gamma_{0}$ is the adjoint, provides a positive definite probability density. It should be emphasized that $J^{\mu}$ is not the only physical observable (bilinear covariant), besides the current density we also have, for instance, in the usual case, bearing in mind the relativistic description of the electron: the invariant length $\sigma=\bar{\psi} \psi$, the pseudo scalar amount $\omega=-i \bar{\psi} \gamma^{5} \psi$, the spin projection in the momentum direction $K^{\mu}=\bar{\psi} \gamma^{\mu} \gamma^{5} \psi$ and the momentum electromagetic density $S^{\mu \nu}=\bar{\psi} i \gamma^{\mu \nu} \psi[2]$. In such a way, we clearly see one of the needs of defining the adjoint structure.

Motivated by the impact of Dirac formulation and the recently new theoretical spin- $(1 / 2)$ particle endowed with mass-dimension-one, which holds a new adjoint structure contrasting with the aforementioned case, we decided to verify the fundamental requirements for the adjoint construction and also we intend to show whether there exists an inherent rigidity or whether it is possible to obtain a new structure for the Dirac adjoint. Following a mathematical procedure, thus, we reach to a new dual structure which holds the same physical content as the previous adjoint definition, however, as a price to be paid, such adjoint structure is "helicity dependent", as can be seen in the context of this work. This paper is organized as follows: in the second section a brief overview about the 
theory of Dirac spinors is introduced. Then, in the third section, we introduce a general prescription to built the dual structure for any spinor, thus, following this recipe we define the new possible dual structure, elucidating the contrasting subtleties. The fourth section is reserved to explore the physical content of the new structure. Lastly, in the fifth section we conclude.

Genesis: basic definitions and a brief overview. - We start our exposition by defining the Dirac spinor as it is commonly presented in the current literature [3]:

$$
\psi(\boldsymbol{p})=\left(\begin{array}{c}
\phi_{R}(\boldsymbol{p}) \\
\phi_{L}(\boldsymbol{p})
\end{array}\right),
$$

the right- and left-handed Weyl spinors transform under a Lorentz boost like

$$
\begin{aligned}
& \phi_{R}(\boldsymbol{p})=e^{+\vec{\sigma} \cdot \hat{\varphi} / 2} \phi_{R}(\mathbf{0}), \\
& \phi_{L}(\boldsymbol{p})=e^{-\vec{\sigma} \cdot \hat{\varphi} / 2} \phi_{L}(\mathbf{0}) .
\end{aligned}
$$

The spinor presented in (1) is an irreducible representation of the Lorentz group with parity symmetry playing a crucial role of linking the $(1 / 2,0) \oplus(0,1 / 2)$ representation spaces, see $[3,4]$. For a direct sum of the representation spaces, the boost generator reads

$$
\kappa=\left(\begin{array}{cc}
-i \boldsymbol{\sigma} / 2 & 0 \\
0 & +i \boldsymbol{\sigma} / 2
\end{array}\right),
$$

such boost generator allows us to connect the rest spinor $\psi(\mathbf{0})$ with the one described by an arbitrary momentum $\psi(\boldsymbol{p})$, as can be seen from

$$
\psi(\boldsymbol{p})=\exp (i \kappa \cdot \hat{\varphi}) \psi(\mathbf{0}),
$$

thus, the Lorentz-transformed single-helicity spinor reads

$$
\psi(\boldsymbol{p})=\sqrt{\frac{E+m}{2 m}}\left(\begin{array}{l}
\left(\mathbb{1}+\frac{p \vec{\sigma} \cdot \hat{p}}{E+m}\right) \phi_{R}(\mathbf{0}) \\
\left(\mathbb{1}-\frac{p \vec{\sigma} \cdot \hat{p}}{E+m}\right) \phi_{L}(\mathbf{0})
\end{array}\right) .
$$

Suppose the components in eqs. (2) and (3) to be under action of the parity symmetry, as a consequence we experience the following relation: $\phi_{R}(\boldsymbol{p}) \leftrightarrow \phi_{L}(\boldsymbol{p})$, due to the fact that the boost generator changes sign, so, the previous statement makes it possible to write

$$
\begin{aligned}
& e^{+\vec{\sigma} \cdot \hat{\varphi} / 2} \phi_{R}(\mathbf{0}) \rightarrow e^{-\vec{\sigma} \cdot \hat{\varphi} / 2} \phi_{R}(\mathbf{0})=\phi_{L}(\boldsymbol{p}), \\
& e^{-\vec{\sigma} \cdot \hat{\varphi} / 2} \phi_{L}(\mathbf{0}) \rightarrow e^{+\vec{\sigma} \cdot \hat{\varphi} / 2} \phi_{L}(\mathbf{0})=\phi_{R}(\boldsymbol{p}) .
\end{aligned}
$$

Imposing to the right- and left-hand components to be eigenstates of the helicity operator, $\vec{\sigma} \cdot \hat{p}$, i.e.,

$$
\vec{\sigma} \cdot \hat{p} \phi_{R / L}^{ \pm}(\mathbf{0})= \pm \phi_{R / L}^{ \pm}(\mathbf{0}),
$$

through the relations above, it becomes easy to define the rest frame spinors up to a phase

$$
\phi_{R}^{+}(\mathbf{0})=\phi_{L}^{+}(\mathbf{0})=\sqrt{m} e^{i \varpi_{1}}\left(\begin{array}{c}
\cos (\theta / 2) e^{-i \phi / 2} \\
\sin (\theta / 2) e^{i \phi / 2}
\end{array}\right),
$$

and

$$
\phi_{R}^{-}(\mathbf{0})=\phi_{L}^{-}(\mathbf{0})=\sqrt{m} e^{i \varpi_{2}}\left(\begin{array}{c}
-\sin (\theta / 2) e^{-i \phi / 2} \\
\cos (\theta / 2) e^{i \phi / 2}
\end{array}\right) ;
$$

in this work we take $\varpi_{1}=\varpi_{2}=0$. As remarked in [5], an important fact concerning the relations in the rest-frame of reference must be highlighted, the derivation of the Dirac equation is only completely correctly accomplished only if one assumes $\phi_{R}^{ \pm}(\mathbf{0})=\phi_{L}^{ \pm}(\mathbf{0})$ for particles and does not neglect $\phi_{R}^{ \pm}(\mathbf{0})=-\phi_{L}^{ \pm}(\mathbf{0})$ for antiparticles, as commonly done in most of the textbooks.

Taking advantage of eq. (9), we find the following set of single-helicity spinors:

$$
\begin{aligned}
& \psi_{1}(\boldsymbol{p})=\sqrt{\frac{E+m}{2 m}}\left(\begin{array}{l}
\left.\left(\mathbb{1}+\frac{p \vec{\sigma} \cdot \hat{p}}{E+m}\right) \phi_{R}^{+}(\mathbf{0})\right) \\
\left.\left(\mathbb{1}-\frac{p \vec{\sigma} \cdot \hat{p}}{E+m}\right) \phi_{L}^{+}(\mathbf{0})\right)
\end{array}\right) \\
& \psi_{2}(\boldsymbol{p})=\sqrt{\frac{E+m}{2 m}}\left(\begin{array}{l}
\left(\mathbb{1}+\frac{p \vec{\sigma} \cdot \hat{p}}{E+m}\right) \phi_{R}^{-}(\mathbf{0}) \\
\left(\mathbb{1}-\frac{p \vec{\sigma} \cdot \hat{p}}{E+m}\right) \phi_{L}^{-}(\mathbf{0})
\end{array}\right) .
\end{aligned}
$$

Looking towards a complete set of eigenspinors of the parity operator, we impose that $\psi_{1}(\boldsymbol{p})$ and $\psi_{2}(\boldsymbol{p})$ satisfy the parity relation $\mathcal{P} \psi(\boldsymbol{p})= \pm \psi(\boldsymbol{p})$, as firstly observed and developed in [5], the operator $\mathcal{P}$ reads

$$
\mathcal{P}=m^{-1} \gamma_{\mu} p^{\mu}
$$

Regardless of that we stablish a complete set of eigenspinors of the parity operator

$$
\begin{aligned}
& \psi_{1}(\boldsymbol{p})=\left(\begin{array}{c}
\Upsilon_{+}\left(p^{\mu}\right) \phi_{R}^{+}(\mathbf{0}) \\
\Upsilon_{-}\left(p^{\mu}\right) \phi_{L}^{+}(\mathbf{0})
\end{array}\right), \\
& \psi_{2}(\boldsymbol{p})=-\left(\begin{array}{c}
\Upsilon_{-}\left(p^{\mu}\right) \phi_{R}^{-}(\mathbf{0}) \\
\Upsilon_{+}\left(p^{\mu}\right) \phi_{L}^{-}(\mathbf{0})
\end{array}\right), \\
& \psi_{3}(\boldsymbol{p})=-\left(\begin{array}{c}
\Upsilon_{+}\left(p^{\mu}\right) \phi_{R}^{+}(\mathbf{0}) \\
\Upsilon_{-}\left(p^{\mu}\right) \phi_{L}^{+}(\mathbf{0})
\end{array}\right), \\
& \psi_{4}(\boldsymbol{p})=\left(\begin{array}{c}
\Upsilon_{-}\left(p^{\mu}\right) \phi_{R}^{-}(\mathbf{0}) \\
\Upsilon_{+}\left(p^{\mu}\right) \phi_{L}^{-}(\mathbf{0})
\end{array}\right),
\end{aligned}
$$

in order to summarize the notation, we have chosen to express the boost factor as

$$
\Upsilon_{ \pm}\left(p^{\mu}\right)=\sqrt{\frac{E+m}{2 m}}\left(1 \pm \frac{p}{E+m}\right)
$$

such spinors are restricted to the following condition:

$$
\begin{aligned}
& \mathcal{P} \psi_{i}(\boldsymbol{p})=+\psi_{i}(\boldsymbol{p}), \quad \text { for } i=1 \text { and } i=2, \\
& \mathcal{P} \psi_{i}(\boldsymbol{p})=-\psi_{i}(\boldsymbol{p}), \quad \text { for } i=3 \text { and } i=4,
\end{aligned}
$$


in view of this observation, we state the following:

$$
\begin{aligned}
& \left(\gamma_{\mu} p^{\mu}-m \mathbb{1}\right) \psi_{i}(\boldsymbol{p})=0, \quad \text { for } i=1 \text { and } i=2, \\
& \left(\gamma_{\mu} p^{\mu}+m \mathbb{1}\right) \psi_{i}(\boldsymbol{p})=0, \quad \text { for } i=3 \text { and } i=4,
\end{aligned}
$$

leading us to conclude that $\psi_{1}(\boldsymbol{p})$ and $\psi_{3}(\boldsymbol{p})$ are related with particles, and the remaining two stand for antiparticles.

The emergence of a new dual structure. - As previously mentioned, the program to be implemented and accomplished in the context of the present work is to analyse the possibility to find a new approach to the dual formulation of the Dirac spinor. Such an approach is taken by the authors as important because recently a new class of spin-(1/2) mass-dimension-one dual helicity fermionic fields was theoretically discovered and was introduced in the literature, see ref. [6], and it shows a new dual structure, contrasting with the previous existing cases, e.g., Majorana and Dirac ones. The mass-dimension-one fields has a slightly different dual structure, it reads

$$
\vec{\lambda}_{h}(\boldsymbol{p})=\left[\Xi(\boldsymbol{p}) \lambda_{h}(\boldsymbol{p})\right]^{\dagger} \gamma_{0},
$$

where $h$ stands for the dual helicity, the operator $\Xi(\boldsymbol{p})$ is indeed to exist, $\Xi^{2}(\boldsymbol{p})=\mathbb{1}$ ensuring an invertible map, and last but not least, it is required that its action on any $\lambda_{h}(\boldsymbol{p})$ yields one of the spinors $\lambda_{h^{\prime}}(\boldsymbol{p})$ from the existing set. Once such requirements are met, this new structure yields a real, non-null and invariant norm for the massdimension-one fermionic field. Faced with this, we are led to search for a possible new structure for Dirac's spinor. The usual formulation of Dirac spinors is well known to lead to a dual structure given by

$$
\bar{\psi}(\boldsymbol{p})=[\mathbb{1} \psi(\boldsymbol{p})]^{\dagger} \gamma_{0}
$$

The questions that arise and remain open are the following: Does the structure presented in (24) fit into Dirac's case? Is the Dirac structure unique? In view of these facts, we are looking for a new Dirac dual structure based on something similar to (24). Henceforth, we will define it as $^{1}$

$$
\vec{\psi}_{i}(\boldsymbol{p}) \stackrel{\text { def }}{=}\left[M(\boldsymbol{p}) \psi_{i}(\boldsymbol{p})\right]^{\dagger} \eta,
$$

where $M(\boldsymbol{p})$ stands for an arbitrary $4 \times 4$ matrix and $\eta$ will be posteriorly fixed. Note that the main difference between the dual structure given in (25) and (26) is the replacement of the identity matrix by $M(\boldsymbol{p})$. Using this prescription, we now evaluate the dual structure for the Dirac spinors given in eqs. (15)-(18). The requirement of a Lorentz-invariant norm translates to the assertion that $\eta$ in (26) must anticommute with the generators of the

\footnotetext{
${ }^{1}$ We choose to define the operator $M(\boldsymbol{p})$ rather than $\Xi(\boldsymbol{p})$ in order to avoid confusion and because it does not necessarily holds the same structure as $\Xi(\boldsymbol{p})$.
}

boosts $(\kappa)$ given in (4), and commute with the generators of the rotations $(\zeta)$, given by

$$
\zeta=\left(\begin{array}{cc}
\boldsymbol{\sigma} / 2 & 0 \\
0 & \boldsymbol{\sigma} / 2
\end{array}\right),
$$

thus

$$
\left\{\kappa_{i}, \eta\right\}=0, \quad\left[\zeta_{i}, \eta\right]=0,
$$

where $i=x, y, z$, a straightforward calculation obeying the constraints in (28) result in $\eta=\gamma_{0}$, in agreement with what previously stated in a general context of spinors in $[7,8]$.

To illustrate the mathematical procedure to evaluate $\vec{\psi}(\boldsymbol{p})$, suppose a spinor corresponding to a particle, then it follows ${ }^{2}$

$$
\phi_{R}(\mathbf{0})=\phi_{L}(\mathbf{0})=\left(\begin{array}{l}
a \\
b
\end{array}\right),
$$

and the operator $M(\boldsymbol{p})$ reads

$$
M(\boldsymbol{p})=\left(\begin{array}{ll}
M_{11} & M_{12} \\
M_{21} & M_{22}
\end{array}\right),
$$

where $M_{i j}$ stands for a $2 \times 2$ matrix composed by $w_{i j}$ elements. Thus, the norm is, in summary, given by

$$
\begin{aligned}
& \vec{\psi}(\boldsymbol{p}) \psi(\boldsymbol{p})= \\
& \left\{\frac{(E+p)}{m}\left(|a|^{2} w_{31}^{*}+a b^{*} w_{32}^{*}+a^{*} b w_{41}^{*}+|b|^{2} w_{42}^{*}\right)\right. \\
& +\frac{(E-p)}{m}\left(|a|^{2} w_{13}^{*}+a b^{*} w_{14}^{*}+a^{*} b w_{23}^{*}+|b|^{2} w_{24}^{*}\right) \\
& +\left(|a|^{2} w_{33}^{*}+a b^{*} w_{34}^{*}+a^{*} b w_{43}^{*}+|b|^{2} w_{44}^{*}\right) \\
& \left.+\left(|a|^{2} w_{11}^{*}+a b^{*} w_{12}^{*}+a^{*} b w_{21}^{*}+|b|^{2} w_{22}^{*}\right)\right\},
\end{aligned}
$$

imposing $\vec{\psi}(\boldsymbol{p}) \psi(\boldsymbol{p})$ to be Lorentz invariant, if we choose to recover the original prescription, the only non-null terms must be $w_{i j}$ for $i=j$, and then we have $M(\boldsymbol{p})=\mathbb{1}$; note that in this case the only remaining components are square-like functions. Nevertheless, our intention is to look towards an alternative method, in such a way, we take advantage of the square-like components that is not on its main diagonal. Regardless of that, the dual by construction allows two possibilities, it thus reads

$$
\vec{\psi}_{i}(\boldsymbol{p})=\left[M_{+}(\boldsymbol{p}) \psi_{i}(\boldsymbol{p})\right]^{\dagger} \gamma_{0}, \quad \text { for } i=1 \text { and } i=3 \text {, }
$$

and

$$
\vec{\psi}_{i}(\boldsymbol{p})=\left[M_{-}(\boldsymbol{p}) \psi_{i}(\boldsymbol{p})\right]^{\dagger} \gamma_{0}, \quad \text { for } i=2 \text { and } i=4,
$$

${ }^{2}$ The authors remark that the same mathematical procedure can be employed to compute the dual structure for antiparticle spinors, however, one cannot neglect the relation $\phi_{R}(\mathbf{0})=-\phi_{L}(\mathbf{0})$, for this specific case. 
the presence of the lower indexes + and - is related with the helicity of the components $\phi_{R / L}$, and it follows that the $M(\boldsymbol{p})$ operator in the matricial form reads

$$
M_{+}(\boldsymbol{p})=\left(\begin{array}{cccc}
0 & 0 & \frac{m}{E-p} & 0 \\
0 & 0 & 0 & \frac{m}{E-p} \\
\frac{m}{E+p} & 0 & 0 & 0 \\
0 & \frac{m}{E+p} & 0 & 0
\end{array}\right),
$$

and

$$
M_{-}(\boldsymbol{p})=\left(\begin{array}{cccc}
0 & 0 & \frac{m}{E+p} & 0 \\
0 & 0 & 0 & \frac{m}{E+p} \\
\frac{m}{E-p} & 0 & 0 & 0 \\
0 & \frac{m}{E-p} & 0 & 0
\end{array}\right) \text {, }
$$

both matrices obey the following properties: $M_{ \pm}^{2}(\boldsymbol{p})=\mathbb{1}$, and $M_{ \pm}^{-1}(\boldsymbol{p})$ exist. As main result, note that for this new construction the dual structure is not unique, which strongly contrasts with the previous case. It should be mentioned that the dual structure depends on the helicity of the components, as can be seen in eqs. (31) and (32); spinors endowed with positive helicity components have an operator $M(\boldsymbol{p})$ different from the one endowed with negative helicity components, this is the price to be paid.

Such new structure provides us a real and non-null Lorentz-invariant norm. Nevertheless, we must point out a fact that has already been discussed, the action of (33) and $(34)$ on $\psi(\boldsymbol{p})$ yields a spinor from the established set, in other words,

$$
\begin{array}{ll}
M_{+}(\boldsymbol{p}) \psi_{i}(\boldsymbol{p})=\psi_{i}(\boldsymbol{p}), & \text { for } i=1 \text { and } i=3, \\
M_{-}(\boldsymbol{p}) \psi_{i}(\boldsymbol{p})=\psi_{i}(\boldsymbol{p}), & \text { for } i=2 \text { and } i=4,
\end{array}
$$

in this case the matrices $M_{+}(\boldsymbol{p})$ and $M_{-}(\boldsymbol{p})$ acting on the spinors behave as an identity matrix.

So, now we are able to write the dual structures in terms of $M_{ \pm}(\boldsymbol{p})$ :

$$
\begin{aligned}
& \vec{\psi}_{1}(\boldsymbol{p})=\left(\Upsilon_{-}\left(p^{\mu}\right) \phi_{R}^{+\dagger}(\mathbf{0}) \Upsilon_{+}\left(p^{\mu}\right) \phi_{L}^{+\dagger}(\mathbf{0})\right), \\
& \vec{\psi}_{2}(\boldsymbol{p})=-\left(\Upsilon_{+}\left(p^{\mu}\right) \phi_{R}^{-\dagger}(\mathbf{0}) \Upsilon_{-}\left(p^{\mu}\right) \phi_{L}^{-\dagger}(\mathbf{0})\right), \\
& \vec{\psi}_{3}(\boldsymbol{p})=\left(\Upsilon_{-}\left(p^{\mu}\right) \phi_{R}^{+\dagger}(\mathbf{0}) \Upsilon_{+}\left(p^{\mu}\right) \phi_{L}^{+\dagger}(\mathbf{0})\right), \\
& \vec{\psi}_{4}(\boldsymbol{p})=-\left(\Upsilon_{+}\left(p^{\mu}\right) \phi_{R}^{-\dagger}(\mathbf{0}) \Upsilon_{-}\left(p^{\mu}\right) \phi_{L}^{-\dagger}(\mathbf{0})\right),
\end{aligned}
$$

thus, we accomplished the procedure of verifying the possibility of obtaining new dual structures, in addition, we have seen that it is possible and without loss of generality we have obtained analogous structures as previously defined. Next step is to analyse their physical contents.

The physics behind the new dual structure. This section is reserved to analyse the physical contents of the new dual structure. The above structure satisfies the orthonormal relations (by construction), so we have

$$
\begin{aligned}
& \vec{\psi}_{i}(\boldsymbol{p}) \psi_{i}(\boldsymbol{p})=+2 m, \quad \text { for } i=1 \text { and } i=2, \\
& \vec{\psi}_{i}(\boldsymbol{p}) \psi_{i}(\boldsymbol{p})=-2 m, \quad \text { for } i=3 \text { and } i=4 .
\end{aligned}
$$

Therefore, it is important to evaluate the spin sums once we found a new dual structure, and then verify if such physical amount is proportional or unitarily connected to the Dirac momentum space wave operator, a slightly lengthy calculation reveals us the following relations:

$$
\begin{aligned}
& \sum_{i=1,2} \psi_{i}(\boldsymbol{p}) \vec{\psi}_{i}(\boldsymbol{p})=\left(\gamma_{\mu} p^{\mu}+m \mathbb{1}\right), \\
& \sum_{i=3,4} \psi_{i}(\boldsymbol{p}) \vec{\psi}_{i}(\boldsymbol{p})=\left(\gamma_{\mu} p^{\mu}-m \mathbb{1}\right) .
\end{aligned}
$$

As a consequence the main result for the new Dirac formulation shows that the spin sums found determines not only the wave operator but also the structure of the FeynmanDyson propagator, leading us to conclude that it matches with the previous established amounts, both formulations are indistinguishable, in other words, the new dual does not affect at any level the fermionic statistics neither the propagator structure. Going further, if one evaluates the physical observables, encoded by the 16 bilinear covariants, which now reads

$$
\begin{aligned}
\sigma & =\vec{\psi} \psi, \quad \omega=-i \vec{\psi} \gamma_{5} \psi, \quad J_{\mu}=\vec{\psi} \gamma_{\mu} \psi, \\
K_{\mu} & =-\vec{\psi} \gamma_{5} \gamma_{\mu} \psi, \quad S_{\mu \nu}=\frac{1}{2} \vec{\psi} i \gamma_{\mu \nu} \psi,
\end{aligned}
$$

one finds exactly the same results already found in [9], regardless of, even replacing $\psi^{\dagger} \gamma_{0}$ by $[M(\boldsymbol{p}) \psi]^{\dagger} \gamma_{0}$ in the bilinear forms given in (45), the result remains unchanged, such spinor belongs to type-2 within Lounesto classification. This leads us to believe that regardless of the dual structure chosen, the physical information contained in the spinors is unchanged.

Final remarks. - The purpose of this work, was to show an alternative approach to construct the Dirac dual structure, following the same program as was recently made for the mass-dimension-one fields proposed in the literature. So, we have shown the complete mathematical procedure to compute the dual structure for any spinor, such program is given by a simple recipe as was shown in details in the second section. Despite the way it is commonly presented, the Dirac dual structure is not unique. We have seen in the context of this paper that we can move towards for a more general and broad approach and build a 
different dual structure, which in turns, is "helicity dependent"; we suppose that this is the price to be paid once we are leaving the original formulation. However, physically and mathematically, such new structure encodes all the same physical information as the previous one.

The authors express their gratitude to Professor Dharam Vir Ahluwalia for careful reading the entire first draft of the manuscript and providing many insightful suggestions. We are also grateful to Professor Julio Marny Hoff da Silva for his hopeful questions and the ensuing discussions. RJBR thanks CAPES for the financial support and CHCV thanks PNPD-CAPES for the financial support.

\section{REFERENCES}

[1] Hoff da Silva J. M., Coronado Villalobos C. H., Bueno Rogerio R. J. and Scatena E., Eur. Phys. J. C, 76 (2016) 563.

[2] Crawford J. P., J. Math. Phys., 32 (1991) 576.

[3] Ryder L. H., Quantum Field Theory, second edition (Cambridge University Press, New York) 1996.

[4] Sperança L. D., Int. J. Mod. Phys. D, 2 (2014) 1444003.

[5] Ahluwalia D. V., EPL, 118 (2017) 60001.

[6] Ahluwalia D. V., Adv. Appl. Clifford Algebras, 27 (2017) 2247.

[7] Ahluwalia D. V., Lee Cheng-Yang and Schritt D., Phys. Lett. B, 687 (2010) 248.

[8] Ahluwalia D. V., Lee Cheng-Yang and Schritt D., Phys. Rev. D, 83 (2011) 065017.

[9] Coronado Villalobos C. H. and Bueno Rogerio R. J., EPL, 116 (2016) 60007. 